\title{
Cavity solitons in a medium-size VCSEL
}

\author{
Etienne Averlant ${ }^{1,2}$, Krassimir Panajotov ${ }^{2}$, Thorsten Ackemann ${ }^{3}$, and Mustapha Tlidi ${ }^{1}$, \\ ${ }^{1}$ Optique Nonlinéaire Théorique, Université Libre de Bruxelles (U.L.B.), CP 231, Campus \\ Plaine, 1050 Brussels, Belgium \\ ${ }^{2}$ Brussels Photonics Team (B-PHOT), Department of Applied Physics and Photonics, Vrije \\ Universiteit Brussel, B-1050 Brussels, Belgium \\ ${ }^{3}$ SUPA and Department of Physics, University of Strathclyde, 107 Rottenrow, Glasgow G4 \\ ONG, Scotland, United Kingdom
}

\begin{abstract}
ABSTRAC
We consider a broad area vertical-cavity surface-emitting laser(VCSEL) subject to optical injection. We experimentally investigate the spontaneous formation of a Cavity Soliton (CS) in a medium size ( $80 \mu \mathrm{m}$ diameter) VCSEL. CSs are generated and erased when sweeping optical injection power and a proper frequency detuning between the master laser and the VCSEL is set.
\end{abstract}

Keywords: VCSEL, nonlinear structures, delay differential equations on spatially extended systems

\section{INTRODUCTION}

Localized Structures often called cavity solitons (CSs) have been the subject to a considerable amount of work during the last 20 years. ${ }^{1,2}$ They have now been found in a wide variety of spatially extended systems in biology, ${ }^{3}$ chemistry, ${ }^{4}$ plant ecology,${ }^{5}$ and optics. ${ }^{6-9}$ These structures in VCSELs have been a subject of an active research in the field of nonlinear optics, due to the maturity of the semiconductor technology and the possible applications of localized structures of light in all-optical delay lines ${ }^{10}$ and logic gates. ${ }^{11}$ Moreover, the fast response time of VCSELs makes them attractive devices for potential applications in all-optical control of light.

Cavity solitons are typically generated using a holding beam to ensure optical bistability of the system, and a writing beam to locally make the system evolve from the lower bistability branch to the upper one and reciprocally. ${ }^{7,10}$ Several experiments have been carried out without a holding beam in different setups. These setups include a frequency selective feedback with ${ }^{12}$ or without ${ }^{13}$ a writing beam, saturable absorption, ${ }^{14}$ amplifiers $^{15}$ and others (see ${ }^{16}$ for a review). Even if these experiments did not require a holding beam, they still required a writing beam, a frequency selective delayed feedback or a saturable absorber. Those options make the CS generation system significantly larger and more complicated. Theoretical works predicted that cavity soliton can exhibits spontaneously a motion in the presence of saturable absorption. ${ }^{17}$ When the pump beam has a circular shape CSs move along the boundary on a circular trajectory. ${ }^{18}$ Cavity solitons can undergo a spontaneous motion under the thermal effects ${ }^{19,20}$ or a regular delay feedback ${ }^{21-24}$

One can also use only a holding beam without a writing beam to generate CSs. They can be turned on and off by sweeping injection current ${ }^{7,8}$ or by varying optical injection power. ${ }^{9}$ The latter method is the one we have chosen for this work. Keeping miniaturization in mind, the diameter of the chosen VCSEL is $80 \mu \mathrm{m}$. This makes it a smaller VCSEL than the ones most often used for CS generation(between $100 \mu \mathrm{m}$ and $150 \mu \mathrm{m}$ diameter), ${ }^{8,12,14,15}$ and a larger VCSEL than the one used in ${ }^{7}(40 \mu \mathrm{m})$. The reason why we did not use such a small aperture VCSEL is that so far, one has only been able to generate in there a single CS, placed exactly in the center of the device. This suggests that boundary conditions are quite important in such a device, which should not be the case when one generates CSs.

The paper is organized as follows: in section 2, we describe the experimental setup and we characterize the solitary and optically injected VCSEL. In section 3, we describe the spontaneous formation of localized structures for different waists of the injection beam, different frequency detunings with respect to the VCSEL and different injection currents. Finally, in section 4, we conclude. 


\section{EXPERIMENTAL SETUP AND VCSEL CHARACTERISTICS}

\subsection{Description of the experimental setup}

The experimental setup used for the generation of two-dimensional localized structures is the one described in. ${ }^{9}$ The setup consists of three main parts. The first part is the injection: the holding beam is provided by a commercial Sacher Lasertechnik TEC100-0960-60 External Cavity Diode Laser (Master) isolated with OFR IO5-TiS2-HP optical isolator (OI). The second part consists of a nonlinear material: we use a $80 \mu \mathrm{m}$ diameter, bottom emitting InGaAs multiple quantum well VCSEL. ${ }^{25}$ The response time scale involved in the space-time dynamics of the material $(\approx \mathrm{ps})$ and carriers $(\approx \mathrm{ns})$ are much faster than the thermal time scale $(\approx \mu \mathrm{s})$. The third part consists of the detection of the VCSEL output: the field emitted by the VCSEL is analyzed using an optical spectrum analyzer (OSA). A CCD camera is used to monitor the near field output profile of the VCSEL. Measurements of the VCSEL optical spectrum and the near field profile as a function of current and optical injection power are performed.

To characterise the standalone VCSEL, we first measure its optical spectrum as a function of current. Close to threshold, most of the optical power is emitted in the vertical polarization direction. The optical spectrum of the solitary VCSEL as a function of injected current in the vertical polarization directions is depicted in Fig. 1; the threshold current and the current-induced thermal red shift are indicated with solid white lines.

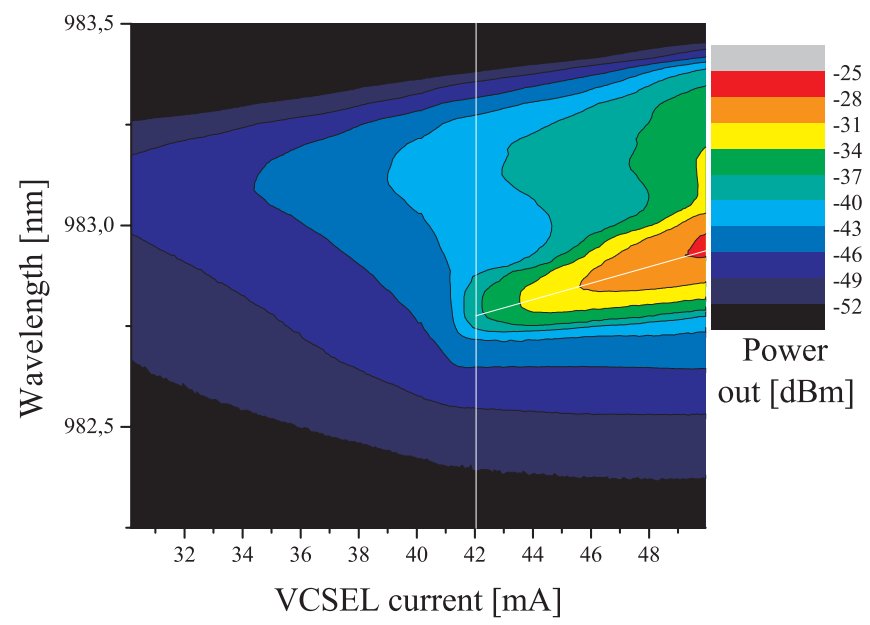

Figure 1. Optical spectrum of the solitary VCSEL as a function of pump current in the vertical polarization. The lasing threshold and current induced thermal red shift have been evidenced by solid white lines. Measures have been performed with the VCSEL kept at $T_{s u b}=25.0^{\circ} \mathrm{C}$.

The near field emission profile of the VCSEL is shown in Fig. 2. This measurement has been obtained for $I=45.0 \mathrm{~mA}>I_{t h}=42.0 \mathrm{~mA}$, where $I_{t h}$ is the lasing threshold at $25^{\circ} \mathrm{C}$. It shows circular standing-wave patterns along the perimeter, sometimes referred to as flower-like, or daisy mode. ${ }^{25}$ It reminds of the one observed in smaller area VCSELs. ${ }^{7}$

\subsection{Optically injected VCSEL}

Finally, we examine the behavior of the VCSEL submitted to optical injection with a polarization direction parallel to the one of the VCSEL. We set the VCSEL current at $I=45.0 \mathrm{~mA}$ and we keep the substrate temperature constant at $T_{s u b}=25.0^{\circ} \mathrm{C}$. Injection locking requires high enough injected power as well as a negative and small enough frequency detuning, which is defined as $\theta=\nu_{i n j}-\nu_{\text {slave }}$, where $\nu_{i n j}$ is the frequency of the injection beam, and $\nu_{\text {slave }}$ is the frequency of the strongest peak in the spectrum of the standalone VCSEL. For this purpose, we need an injection wavelength greater than 982.91nm. Optical spectra are shown in Fig. 3. These spectra have been obtained for two different values of the optical injection power. When the injection beam power is $P_{i n j}=850 \mu \mathrm{W}$, the VCSEL is frequency pulled towards the master laser frequency which is indicated by a short vertical arrow, as shown in Fig. 3(a). The near field emission profile changes, even though the flower-like 
Figure 2. Solitary VCSEL near field profile in the transverse plane, obtained for $I=45.0 \mathrm{~mA}$ and $T_{s u b}=25.0^{\circ} \mathrm{C}$. Black corresponds to high optical power whereas white corresponds to low optical power.

mode has not disappeared from the emitting surface of the VCSEL. In this case, there is no injection locking of the VCSEL. However, for an injection power of $P_{i n j}=2.04 \mathrm{~mW}$, the VCSEL is locked to the master laser as shown in Fig. 3(b).
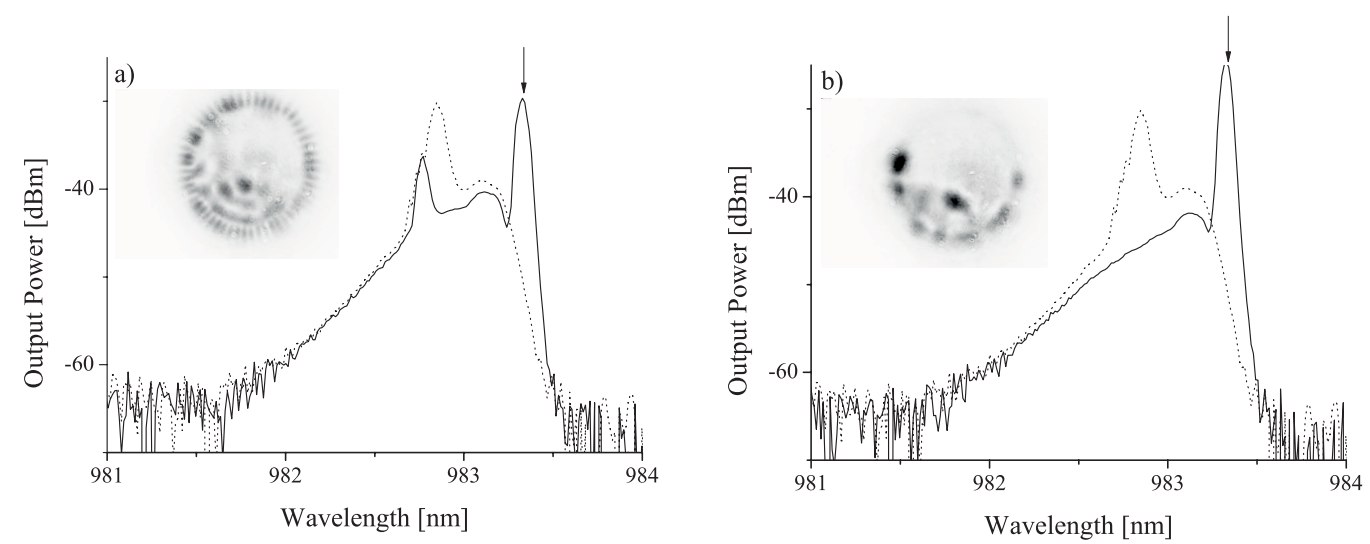

Figure 3. Dashed lines: optical spectra of the free running VCSEL obtained for $I=45.0 \mathrm{~mA}$ and $T_{\text {sub }}=25.0^{\circ} \mathrm{C}$. Solid lines: optically injected VCSEL with $\lambda_{i n j}=983.24 \mathrm{~nm}$ (indicated by a vertical arrow) and injection power of $(\mathrm{a}) P_{i n j}=850 \mu \mathrm{W}$ and (b) $P_{i n j}=2.04 \mathrm{~mW}$. The insets are near field images of the optically injected VCSEL.

\section{SPONTANEOUS FORMATION OF CAVITY SOLITONS IN AN OPTICALLY INJECTED VCSEL}

After the characterization of the VCSEL, we investigate the formation of two-dimensional CSs for different values of detuning and different beam waists. All experimental measurements have been performed when the VCSEL operated in an injection locked regime as in Fig. 3(b).

We first investigated CSs bistability with the injection power for a fixed value of the detuning parameter $\theta=-174 \mathrm{GHz}$ and for a fixed value of the injection beam waist of $100 \mu \mathrm{m}$. The latter is defined as the radius of the smallest circle containing half of the injection beam power when it encounters the VCSEL. The experimental results are summarized in the bistable curve in Fig. 4. The VCSEL output power as a function of the injected beam power, which is shown in this figure, undergoes a bistable behavior between a single CS (i) and a two CSs (ii) states, as shown in Fig. 4. The experimental procedure to obtain CSs consists of increasing the injection power, and, just as the locking region is reached, a single CS appears. Then, as we further increase the optical injection power, we observe transition from a single CS state towards a two-CSs state. This behavior corresponds 
to a spontaneous switching on. To realise the switching off, we decrease the injection power. The two CSs persist until the system reaches the switching down point, over which the system relaxes to the single peak state. The density plot of both 1-CS and 2-CSs near field are recorded by using a CCD camera. Cross sections of the single and the two-CSs states near-field are shown in Fig.5. A fundamental characteristic of CS is that it has an

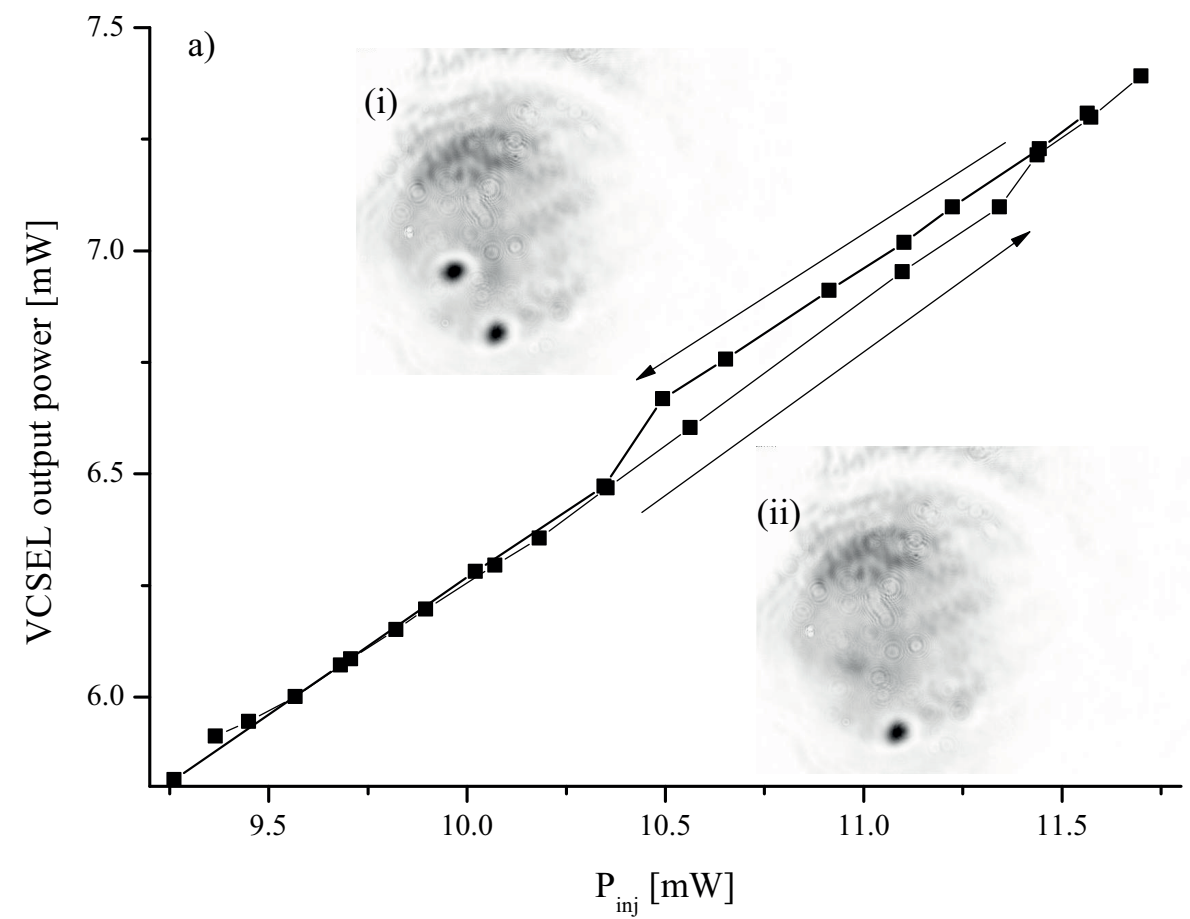

Figure 4. Power emitted by the VCSEL as a function of the optical injection power for $\theta=-174 \mathrm{GHz}$ and a beam waist of $100 \mu \mathrm{m}$. The insets (i) and (ii) respectively represent near field profiles on the higher and lower branch of the hysteresis.
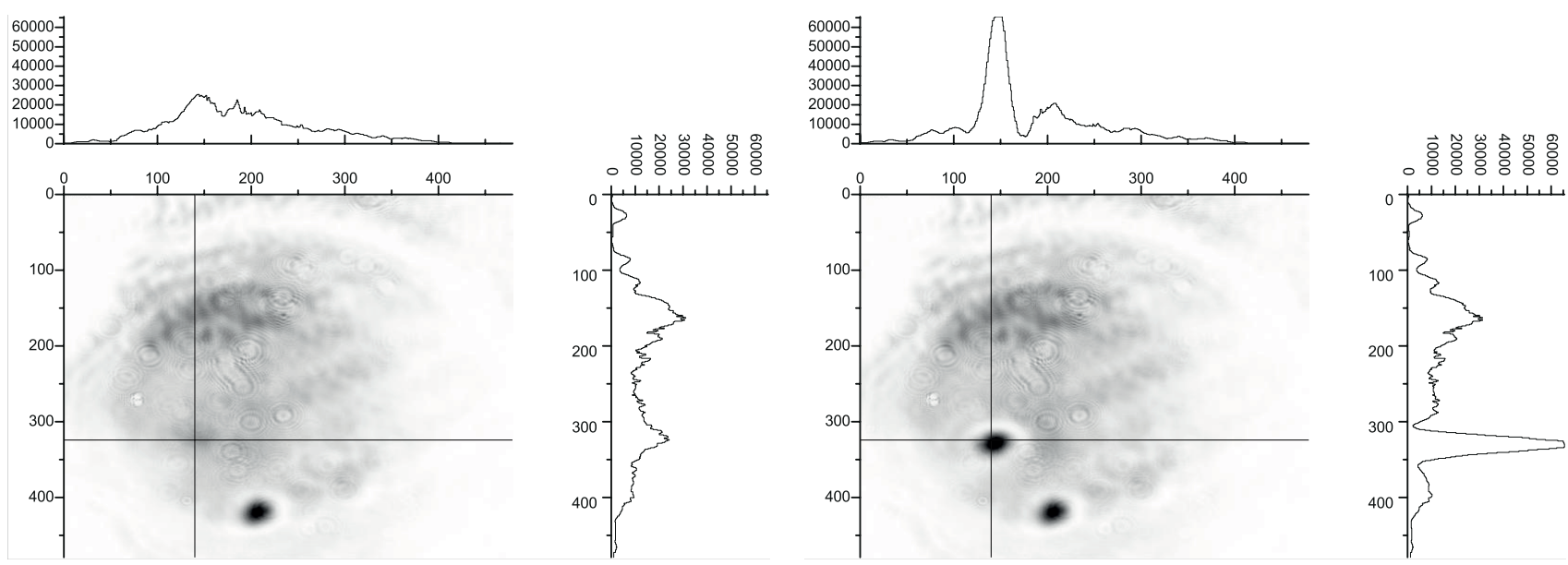

Figure 5. One-dimensional profiles on crossed lines in the near field of the VCSEL for (left) the inset (i) of Fig.4 and (right) the inset (ii) of the latter. Both axis on the pictures are the coordinates of each pixel on the CCD captor, whereas the $y$ axis on each profile is the gray level.

oscillatory tail, which decays exponentially with the distance to the center of the CS. 


\section{CONCLUSIONS AND PERSPECTIVES}

To conclude, we report experimental evidence of spontaneous formation of CSs in a $80 \mu$ m diameter VCSEL submitted to optical injection. Different detunings between the frequencies of the injection beam and the VCSEL have been investigated, as well as different beam waists. This behavior occurs by varying the optical injection power, without needing a writing beam: the system evolves spontaneously from the lower branch of the hysteresis to the upper one and reciprocally.

The analysis of local polarization dynamics ${ }^{7}$ in the transverse plane of the $80 \mu \mathrm{m}$ VCSEL, the occurrence of polarization patterns and possibility the realization of LSs between two polarization modes can be of interest for continuation of this work. Studies of vector solitons in polarization will be theoretically carried out using the spin-flip model of VCSELs, and implemented experimentally.

\section{Acknowledgments}

M.T. received support from the Fonds National de la Recherche Scientifique (Belgium). This research was partially supported by EU FP7 ICT FET Open HIDEAS and by the Interuniversity Attraction Poles program of the Belgian Science Policy Office, under grant IAP P7-35 photonics@be.

\section{REFERENCES}

[1] M. Tlidi et al., Phys. Rev. Lett. 73, 640 (1994); A.J. Scroggie et al., Chaos Solitons and Fractals, 4, 1323 (1994).

[2] L.A. Lugiato, Chaos, Solitons \& Fractals, 4, 1251 (1994).

[3] J.D. Murray, Mathematical Biology, 3de ed. (Springer, Berlin, 2003).

[4] V. K. Vanag et al., Nature 406, 389 (2000); I. Lengyel and I. Epstein, Proc. Natl. Sci. U.S.A. 89, 128301 (2004); T. Kolokolnikov and M. Tlidi, Phys. Rev. Lett. 98, 188303 (2007); M. Tlidi, G. Sonnino, and M. Bachir, Phys. Rev. E 86, 045103 (2012).

[5] O. Lejeune et al., Phys. Rev. E 66, 010901(R) (2002); E. Meron et al., Chaos, Solitons and Fractals, 19, 367 (2004); M. Rietkerk et al., Science 305, 1926 (2004); E. Meron et al., Chaos, 17, 037109 (2007); M. Tlidi et al., Lecture Notes in Physics, 751, 381 (2008); E. Sheffer et al. J. Theor. Biol., 273, 138 (2011).

[6] V.B. Taranenko, K. Staliunas, and C.O. Weiss, Phys. Rev. A 56, 1582 (1997); V.B. Taranenko et al., Phys. Rev. Lett. 81, 2236 (1998).

[7] X. Hachair et al., Phys. Rev. A 72, 013815 (2005); D. Bajoni et al., Phys. Rev. Lett. 101, 266402 (2008); P. Genevet et al., Phys. Rev. Lett. 101, 123905 (2009)

[8] X. Hachair, G. Tissoni, H. Thienpont, and K. Panajotov, Phys. Rev. A 79, 011801(R) (2009).

[9] E. Averlant et al., Optics Express, 22, 762 (2014).

[10] F. Pedaci, S. Barland, E. Caboche, P. Genevet, M. Giudici, J. R. Tredicce, T. Ackemann, A. J. Scroggie, W. J. Firth, G. L. Oppo, G. Tissoni, and R. Jager, Appl. Phys. Lett. 92, 011101 (2008).

[11] A. Jacobo, D. Gomila, M. A. Matáas, and P. Colet, New J. Phys. 14 , 013040 (2012).

[12] Y. Tanguy, T. Ackemann, W. J. Firth, and R. Jager, Phys. Rev. Lett. 100, 013907 (2008).

[13] N. Radwell and T. Ackemann, IEEE J. Quantum Electron. 45, 1388 (2009).

[14] P. Genevet, S. Barland, M. Giudici, and J. R. Tredicce, Phys. Rev. Lett. 101, 123905 (2008).

[15] Y. Menesguen, S. Barbay, X. Hachair, L. Leroy, I. Sagnes, and R. Kuszelewicz, Phys. Rev. A 74, 023818 (2006).

[16] S. Barbay, R. Kuszelewicz, and J. R. Tredicce, Advances in Optical Technologies, Volume 2011 (2011), Article ID 628761, 23 pages, doi:10.1155/2011/628761.

[17] S. V. Fedorov, A. G. Vladimirov, G. V. Khodova, and N. N. Rosanov, Phys. Rev. E 61, 5814 (2000).

[18] F. Prati, G. Tissoni, L. A. Lugiato, K. M. Aghdami, and M. Brambilla, Eur. Phys. J. D 59, 73-79 (2010).

[19] L. Spinelli, G. Tissoni, L. A. Lugiato, and M. Brambilla, Phys. Rev. A 66 , 023817 (2002).

[20] A. J. Scroggie, J. M. McSloy, and W. J. Firth, Phys. Rev. E 66, 036607 (2002).

[21] M. Tlidi, A.G. Vladimirov, D. Pieroux, and D. Turaev, Phys. Phys. Rev. Lett. 103, 103904 (2009).

[22] M. Tlidi, E. Averlant, A. Vladimirov, and K. Panajotov, Phys. Rev. A 86, 033822 (2012). 
[23] K. Panajotov and M. Tlidi, Eur. Phys. J. D 59, 67 (2010).

[24] A. Pimenov, A. G. Vladimirov, S. V. Gurevich, K. Panajotov, G. Huyet, and M. Tlidi, Phys. Rev. A 88, 053830 (2013).

[25] M. Grabherr, M. Miller, R. Jager, R. Michalzik, U. Martin, H. J. Unold, and K. J. Ebeling, IEEE J. Sel. Top. Quantum Electron. 5, 495 (1999). 